\title{
Experimental investigation of temperature fields in a synthetic jet
}

\author{
Petra Dančová ${ }^{1,2, a}$, Roman Doleček ${ }^{1,3}$, and Vít Léd1 ${ }^{1,3}$ \\ ${ }^{1}$ Technical University of Liberec, Studentská 2, 46117 Liberec 1, Czech Republic \\ ${ }^{2}$ Institute of Thermomechanics, AS CR, v.v.i., Dolejškova 5, 18800 Prague 8, Czech Republic \\ ${ }^{3}$ TOPTEC, Institute of Plasma Physic AS CR, v.v.i., Sobotecká 1660, 51101 Turnov, Czech Republic
}

\begin{abstract}
The paper deals with the measurement of temperature fields in a synthetic jet. This type of experiment presents several challenges; therefore, to ensure the best precision possible, two experimental methods were used - digital holographic interferometry (DHI) and thermo-anemometry in constant current mode as an auxiliary method to verify DHI.
\end{abstract}

\section{Introduction}

A synthetic jet (SJ) is generated by the periodic motion of an actuator oscillating membrane. A SJ is synthesized by the interactions within a train of vortex rings or counterrotating vortex pairs in axisymmetric or two-dimensional geometry; see Smith and Glezer [1]. Vortex rings are formed at the lip of the orifice (see Figure 1). These rings move in $y$ direction at a velocity high enough to prevent interaction with the suction in the orifice. It has been previously observed that an SJ far enough from the orifice assumes the characteristics of a conventional steady jet. This is caused by the development and dissipation of vortexes. One of the main advantages of a $\mathrm{SJ}$ is that the time-mean mass flux of the oscillatory flow in the orifice is zero; hence, the other common description for a SJ, a zero-net-mass-flux (ZNMF) jet. A ZNMF eliminates the need for a blower and piping for the fluid inlet. Though the SJ actuator works with a ZNMF at the orifice, the momentum of the resultant SJ at a specific distance from the lip along $y$ is non-zero [1-3].

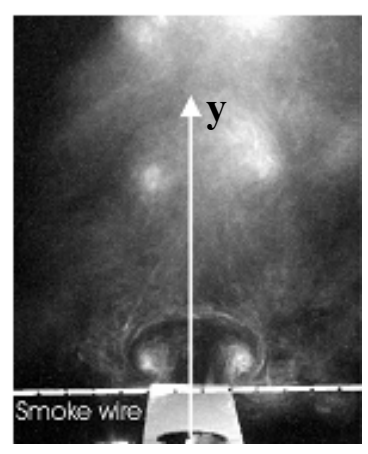

Figure 1. Creation of vortex rings by fluid extrusion from the cavity of an actuator. Results of a "smoke wire" visualization, [4].

$\bar{a}$ Corresponding author: petra.dancova@tul.cz
SJs have many promising applications, typically in flow control (jet vectoring, active flow control in external and internal aerodynamics), mixing, and heat- and masstransfer treatment, e.g. [5-8].

The main parameters of the SJ used in the experiment were:

The mean time orifice velocity:

$$
U_{0}=\frac{1}{\tau} \int_{0}^{\tau / 2} u_{0}(t) d t
$$

where $\tau$ is the time period, i.e. $\tau=1 / f$ and $f$ is the frequency, and $u_{0}(\mathrm{t})$ represents the periodical axial orifice velocity.

And the actuator working (resonance) frequency:

$$
f=\frac{1}{2 \pi \cdot C} \frac{D}{D_{\mathrm{m}}} \sqrt{\frac{k_{\mathrm{p}}}{\rho L_{\mathrm{e}}}} .
$$

At the resonance frequency, the SJ actuator works at its highest efficiency. During the working cycle of a SJ actuator, the potential energy of the actuator membrane is transformed into kinetic energy of the fluid in the orifice. In eq. (2), $D$ and $D_{\mathrm{m}}$ are the actuator orifice diameter and diameter of the oscillating membrane respectively, $k_{\mathrm{p}}$ $\left(\mathrm{N} \cdot \mathrm{m}^{-3}\right)$ is the stiffness of the membrane, $\rho$ is the density of working fluid, $L_{\mathrm{e}}$ is equivalent length, and $C$ is the constant, which corrects to the deformed membrane shape.

Non-dimensional temperature $T^{*}$ is defined as:

$$
T^{*}=\left(T-T_{a}\right) /\left(T_{0}-T_{a}\right)
$$


where $T$ is the temperature of the fluid, $T_{\mathrm{a}}$ is the ambient temperature, and $T_{0}$ is the average temperature at the nozzle.

\section{Experimental setup and methods}

Figure 2 shows the arrangement of the experiment. The SJ actuator consisted of a sealed cavity, which was equipped with an emitting orifice oriented vertically upwards (diameter $D=5 \mathrm{~mm}$ ) and a pair of ARN-100$10 / 4$ loudspeakers used as the oscillating membrane. These loudspeakers were running in opposite directions. The diameter of the loudspeakers was $94 \mathrm{~mm}$, their nominal electrical resistance $4 \Omega$. An OMEGALUX CIR$10301 / 240 \mathrm{~V}$ cartridge heater was equipped with a K-type thermocouple and placed into the holder tube inside the actuator cavity. During experiments, it was possible to control the temperature $T_{\mathrm{c}}$ of the heating cartridge surface up to $200{ }^{\circ} \mathrm{C}$ via a connected PID regulator. The uncertainty of the temperature of the wall was less than $0.2 \mathrm{~K}$ during each experiment. The experiments used air as working fluid.

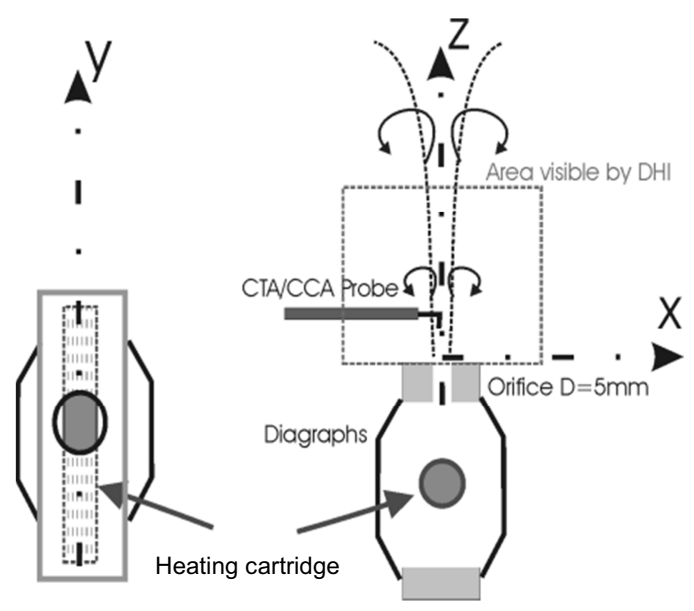

Figure 2. Schematic view of experimental arrangement.

\subsection{Digital holographic interferometry}

Digital holographic interferometry (DHI) is a contactless, non-invasive, and whole field measurement experimental technique. In this study, DHI was used to measure temperature fields in air generated by a heated SJ. DHI principles are described in detail in works [9-12]. In brief, this method is based on two holograms of wave fronts recorded by a digital camera. The first hologram $h_{1}$ corresponds with an initial state of the temperature field, while the second one $h_{2}$ corresponds to its subsequent state. If these two holograms are captured, one can numerically reconstruct their optical fields $U_{1}(m . n)$ and $U_{2}(m . n)$ in the image plane by means of the Sommerfeld formula

$$
\begin{aligned}
& U(n \Delta x, m \Delta y)=e^{j \pi \lambda d\left(\left(\frac{n \Delta x}{N \Delta \xi}\right)^{2}+\left(\frac{m \Delta y}{M \Delta \eta}\right)^{2}\right)} \times \\
& \times \sum_{k=1}^{N} \sum_{l=1}^{M} h(k \Delta \xi, l \Delta \eta) r^{*}(k \Delta \xi, l \Delta \eta) e^{\frac{j \pi}{\lambda d}\left((k \Delta \xi)^{2}+(l \Delta \eta)^{2}\right)} e^{-j 2 \pi\left(\frac{k n}{N}+\frac{l m}{M}\right)}
\end{aligned}
$$

where $j=\sqrt{-1}, \lambda$ is the wavelength of the light used and $d$ is distance between the CCD and the temperature field. $N \times M$ represents the number of pixels in a perpendicular direction and the pixel center-to-center distance of the CCD used is $\Delta \xi \times \Delta \eta$. The symbol $r^{*}$ represents the complex amplitude of the conjugated reference wave.

The phase distribution of both reconstructed fields were obtained using

$$
\begin{aligned}
& \phi_{1}(m, n)=\arctan \frac{\operatorname{Im}\left(h_{1}(m, n)\right)}{\operatorname{Re}\left(h_{1}(m, n)\right)}, \\
& \phi_{2}(m, n)=\arctan \frac{\operatorname{Im}\left(h_{2}(m, n)\right)}{\operatorname{Re}\left(h_{2}(m, n)\right)}
\end{aligned}
$$

The interference phase $\Delta \varphi(n, m)$ relates to the temperature field variation and can be determined in a point wise manner by means of a modulo $2 \pi$ subtraction

$$
\Delta \varphi=\left\{\begin{array}{llc}
\varphi_{1}-\varphi_{2} & \text { if } & \varphi_{1} \geq \varphi_{2} \\
\varphi_{1}-\varphi_{2}+2 \pi & \text { if } & \varphi_{1}<\varphi_{2}
\end{array} .\right.
$$

The desired result of this experiment was to gather accurate measurements of the temperature distribution in the SJ. The value of this physical quantity was determined by the effect it had on the refractive index field. The key quantity was the density $\rho$ of the gas. Its relation to the refractive index $n$ is given by the GladstonDale equation:

$$
n-1=K \rho,
$$

where $K$ is the Gladston-Dale constant and $\rho$ is the density, which are the properties of the gas, and $n$ is the refractive index. The density $\rho$ of the gas can be calculated from the pressure $P$, the molecular weight $M$, and the absolute temperature $T$ via the ideal gas equation:

$$
\rho=\frac{M P}{R T} .
$$

Figure 3 shows the configuration of the DHI setup used for the SJ temperature field experiments described in this paper. Previous experiments $[12,13]$ demonstrated the effectiveness of an interferometric setup based on a Twymann-Green interferometer. In this setup, the light traveled through the phase object twice, which doubles the sensitivity of the setup. As shown in figure 3, a 150- 
$\mathrm{mW}$ laser beam $(\mathrm{Nd} 3+$ :YAG) was divided by a polarizing beam splitter (BS1) equipped with $\lambda / 2$ plates. After division, both beams were filtered by spatial filters (SF) and then collimated by collimating objectives (CO). Apertures placed in front of the collimating objectives ensured a final beam diameter of $50 \mathrm{~mm}$. After collimation, beam " 2 " entered beam splitter BS2. One part of the beam was reflected by the beam splitter while the second part traveled through the measured object and perpendicularly impinged on mirror M3, where it was reflected and traveled through the measured object once more. This is the reason why the interferometer had double sensitivity. Then, once again in beam splitter BS2, part of the light was reflected towards the CCD sensor while the second part continued in the direction of the collimating objective $\mathrm{CO} 2$. After collimation, the beam tagged as "1" (the reference beam) was reflected by mirror M2 towards the CCD sensor and traveled through beam splitter BS2; both beams impinged the CCD sensor from slightly different directions. The camera and the pulsatile jet were synchronized. However, the setup was complicated to adjust and was not very light efficient; energy was lost mainly in the beam splitter BS2.

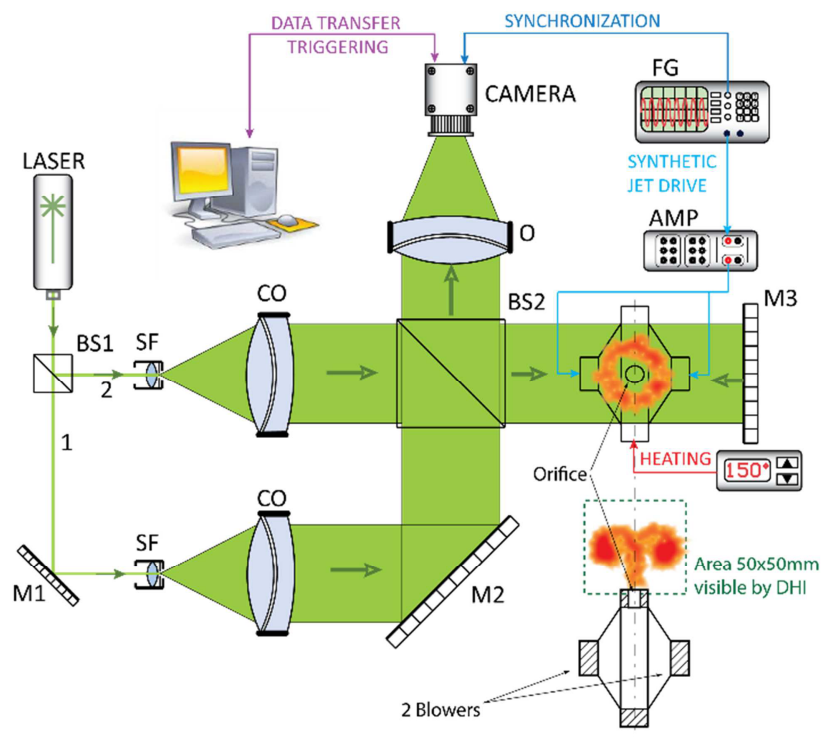

Figure 3. Setup for DHI with double sensitivity (BS - beam splitter, M - mirror, SF - spatial filter, CO - collimating objective, $\mathrm{O}$ - focusing objective, $\mathrm{FG}$ - function generator).

The principle of thermo-anemometry in constant current mode is described in detail in [14]. Briefly, the current through the probe wire is maintained at a constant value. Probe wire temperature $T_{\text {wire }}$ varies with the velocity and temperature of the flowing fluid. Changing the probe wire temperature causes a change in the electrical resistance and voltage $E_{\text {wire }}$ of the probe wire.

\subsection{Synchronization of the experiment}

As mentioned above, a SJ exhibits periodic motion. One part of the actuating period represents suction into, and the second half extrusion from, the actuator. To determine the phase of flow, it was necessary to synchronize a SJ actuator with the experimental setup. The SJ actuator worked with resonance frequency of 15 $\mathrm{Hz}$ (calculated from eq. (2)). A 5MP AVT Stingray camera was used in our experiment for hologram recording. The holograms were cropped to 2048 x 2048 pixels. Unfortunately, 5MP cameras are still quite slow. The maximum frame rate of an AVT Stingray camera at this resolution is approximately 6 FPS. To achieve a good temporal resolution, we would need at least 300 FPS at a frequency of $15 \mathrm{~Hz}$ (20 samples per period). In the periodic behavior of the pulsatile jet operation we could presume the phenomenon as a quite coherent (every puff is similar). Due to this assumption, we were able to synchronize camera capture with a specifically selected time within a period (we were able to capture certain phases of the phenomenon). However, the frame rate of the camera was much slower than the frequency of the phenomenon. Thus, it was necessary to ensure that the camera waited until the buffer was empty before another capture was taken in a different phase of the phenomenon. Some periods were usually skipped. This approach had many benefits, the most obvious of which was that a few frames with the same phase could be captured and later averaged. The basic principle behind the synchronization method is shown in figure 4 .

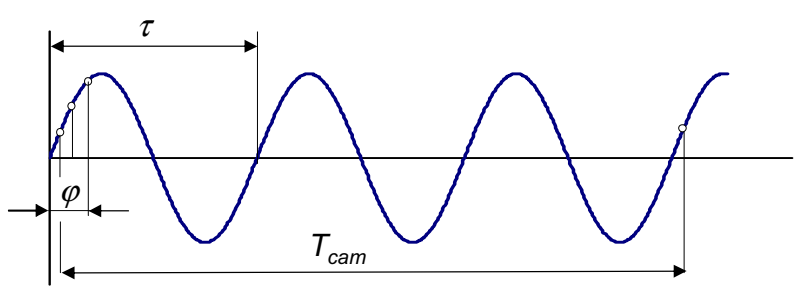

(a)
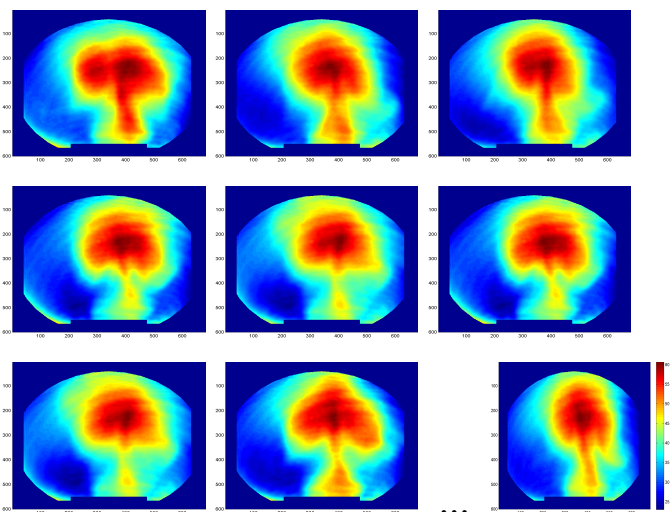

(b)

Figure 4

(a) Principle behind the synchronization method, $\varphi$ is the measured phase, $T_{\mathrm{CAM}}$ is the period given by the frame rate of the camera; the sinusoidal curve denotes the periodic development of the measured phenomenon.

(b) Evaluated 2D temperature fields in the same phase of different periods of the SJ. 


\section{Results}

Mentioned above, the resonance frequency was determined as $15 \mathrm{~Hz}$. On this frequency whole experiments were carried out. In Figure 4b), the outcome of the triggering process of a coherent SJ phenomenon and the digital camera is shown. It verifies the previous assumption of the coherence of the SJ phenomenon. Figure $4 \mathrm{~b}$ ) shows the reconstructed $2 \mathrm{D}$ temperature fields as they correspond to the same relative times but in different periods of the repeated phenomenon. The differences between the reconstructed fields are easy to see. These uncertainties were caused by the physical principle of the phenomenon and by random fluctuation. On the other hand, one can capture sufficient data that corresponds to the same relative time and then average the results.

Figure 5 shows a comparison of the 2D temperature fields acquired by DHI (left side of each picture) and by thermo-anemometry in constant current mode (right side of each picture). The pictures correspond to different phases during the cycle $t / \tau$. The development of the 2D structure is clearly visible. Figure 5 illustrates the ability of DHI to visualize 2D temperature fields. The position and development of large coherent structures, which puff from the orifice, are evident. It must be noted that in $2 \mathrm{D}$ cases, DHI integrates the phase change along the entire path of the rays going through the measured area (through the $y$ axes), and the results must be presented as the average temperature in the $y$ direction. The Abel transformation could be used to partially solve this problem. However, the application of a tomographic approach is sufficient.

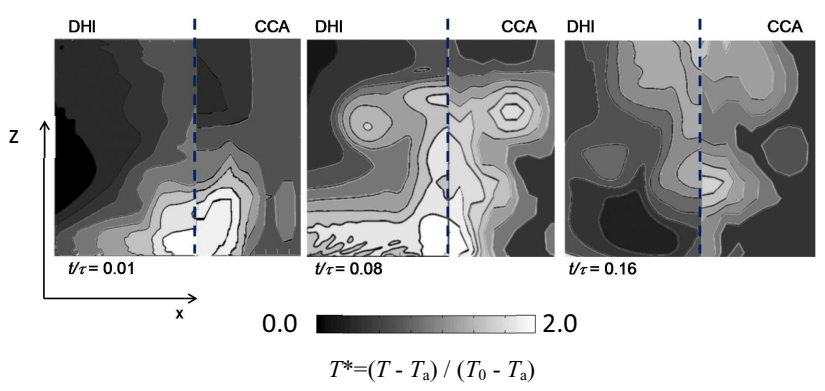

Figure 5. Temperature field above the jet. Images at different $\mathrm{t} / \tau$ achieved by digital holographic interferometry (DHI) and by thermo-anemometry in constant current mode (CCA).

Figure 6 illustrates the comparison of temperatures achieved by DHI and by thermo-anemometry in constant current mode in a stream-wise direction. The results from DHI show the average temperature in the $y$ direction. The thermo-anemometry results were achieved at $y=0$.

\section{Conclusions}

This paper combines the results of measurements from temperature field research of the synthetic jet using two methods - DHI and thermo-anemometry in constant current mode. Paper describes also the explanation of synchronization between the synthetic jet actuator and the measuring system.

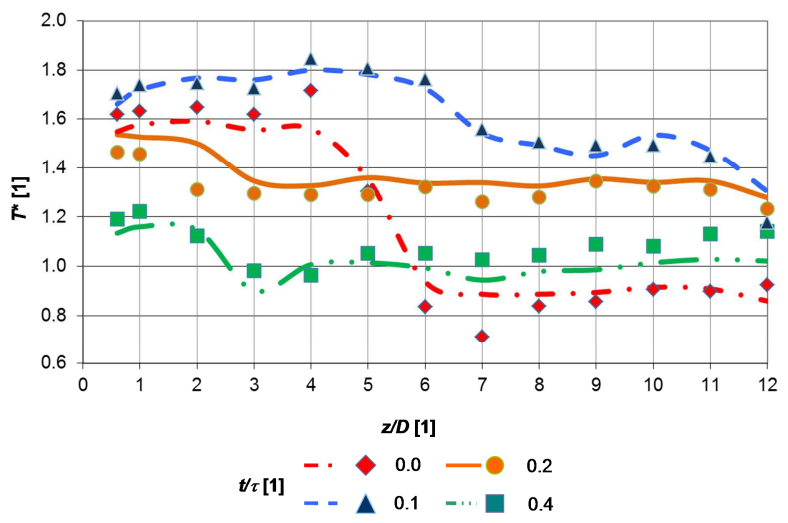

Figure 6. Comparison of temperatures ascertained via DHI (solid lines) and via thermo-anemometry (points).

The above-mentioned tomographic techniques require a large number of different projections, which are usually acquired from several sensors. In another paper [11], the authors use this nontraditional approach with one triggered camera and periodic phenomenon to acquire sufficient data for a tomographic reconstruction of the temperature field.

Acknowledgements We gratefully acknowledge the support the ESF operational program "Education for Competitiveness" in the Czech Republic as part of the project "Support of engineering of excellent research and development teams at the Technical University of Liberec" No. CZ.1.07/2.3.00/30.0065. We would also like to thank the SGS 21000 project and the Ministry of Education, Youth and Sports of the Czech Republic for Project No. CZ.1.05/2.1.00/03.0079: Research Center for Special Optics and Optoelectronic Systems.

\section{References}

1. B.L. Smith, A. Glezer, Phys. Fluids 10 (1998), pp. 2281-2297

2. J.E. Cater, J. Soria, J. Fluid Mech. 472 (2002), pp. $167-200$

3. A. Glezer, M. Amitay, Annu. Rev. Fluid Mech. 34 (2002), pp. 503-529

4. P. Dančová, Analysis of the Synthetic Jet, Master thesis (2006)

5. B.L. Smith, A. Glezer, J. Fluid Mech. 458 (2002), pp. 1-34

6. M. Amitay, D. R. Smith, V.Kibens, D. E. Parekh, A. Glezer,: AIAA J. 39, 3 (2001), pp. $361-370$

7. M. Ben Chiekh, J.C. Bera, M. Sunyach,. J. Turbulence 4, 032 (2003)

8. Z. Trávníček, A.I. Fedorchenko, A.B. Wang, Sensors and Actuators A 120, 1 (2005), pp. 232-240

9. T. Kreis, Handbook of Holographic Interferometry: Optical and Digital Methods (Berlin: Wiley, 2004) 
10. V. Lédl, T. Vít, R. Doleček, P. Psota, EPJ Web of Conferences 25, 02014 (2012)

11. R. Doleček, P. Psota, V. Lédl, T. Vít, V. Kopecký, EPJ Web of Conferences 67, 02021 (2014)

12. T. Vít, V. Lédl, AIP Conference Proceedings 1281 (2010), pp. 135-138

13. R. Doleček, V. Lédl, V. Kopecký, P. Psota, J. Václavík, T. Vít, Proc.of the international conference Experimental Fluid Mechanics 2009 (2009), pp. 25-33

14. H.H. Bruun, Hot wire anemometry, Oxford University Press (1995) 